\title{
Postion angle jumps in pulsar linear polarization
}

\author{
R.X. Xu, G.J. Qiao \\ Department of Geophysics, Peking University, Beijing 100871, China
}

J.L. Han

Beijing Astronomical Observatory, CAS, Beijing 100080, China

\begin{abstract}
The position angle (PA) behaviour of linear polarization of pulsar emission is simulated after considering the relative retardation between the core and conal components, which are believed to be generated from different emission altitudes. The PA will jump $90^{\circ}$ at some points where the complete depolarization occurs.
\end{abstract}

The diversity of PA behavior of linear polarization observed from a large number of pulsars stands in stark contrast to the widely adopted simple and systematic pattern of rotation vectors in the rudimentary magnetic pole model (Radhakrishnan $\&$ Cooke 1969). It is often found that the PA jumps in a very small range of rotation longitude, sometimes up to $90^{\circ}$.

With polarization information, core and conal components are identified from mean pulsar profiles (Rankin 1983; Lyne and Manchester 1988). It is suggested that core emission comes from lower altitude above the neutron star surface. Qiao $(1988,1992)$ suggest that the Inverse Compton Scattering (ICS) process can be responsible for the pulsar radio emission. The core, inner cone and outer cone emission components are incoherently emitted at different heights above the neutron star surface (Qiao et al. 1992). Here we show by simulations that superposition of these emission beams will depolarize the observed mean profiles and the PA will jump at some points if the relative aberration and retardation effects are considered.

In our simulation, we assume:

(1). The core, inner cone and outer cone emission components are emitted at different heights and the apparent position of the emission beam of these components will relatively shift in longitude. In fact, we are not concerned about the emission mechanism;

(2). The emissions from different components are incoherent;

(3). For each component, the polarization plane behaves as in the well known rotation vector model;

(4). The intensity distribution of core emission is a Gaussian, while that of the conal emission ia a torus with a transection of a Gaussian.

The "observed" Stokes' parameters are the sum of every component, so $I=$ $\Sigma I_{i}, Q=\Sigma Q_{i}, U=\Sigma U_{i}$, and $V=\Sigma V_{i}$, where $i=1,2,3$ denotes the core, inner cone and outer cone respectively. In the simulation for the simplest case shown in Fig.1, only linear polarized one core and one cone are considered, and the circular polarization is not. Owing to depolarization, we find that at some points, 
so-called "singular points" the linear polarization intensity $L$ can be to zero (completely depolarized), $L=\sqrt{Q^{2}+U^{2}}=0$, because the emission intensities of two components are equal but polarization planes are perpendicular. It is not difficult to verify that the "observed" PA will jump $90^{\circ}$ when the line of sight travels across one of these sigular points.

It should be emphasized that such a simulation is valid for all emission mechanism, as long as the core and conal emission comes from different emission altitudes and has a relative retardation and aberration.

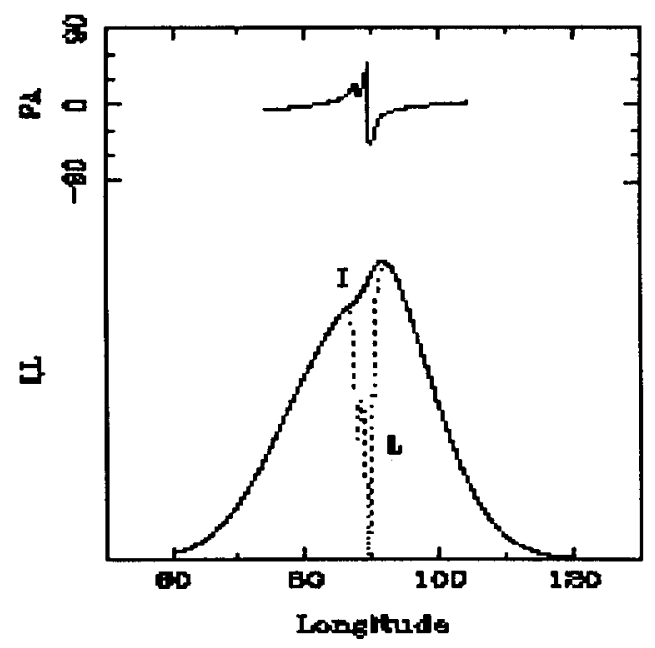

Figure 1. The simulated integrated pulse profile. The PA jumps, somtimes up to $90^{\circ}$, in the very small range of longitude. The situation can be found from, for instance, PSR B1604-00 (Rankin 1988), PSR B1601-52 (Qiao et al. 1995), PSR B1556-44 (Wu et al. 1993). At this "singular" point of pulsar emission beam, strong depolarization occurs.

Acknowledgments. We thank the National Nature Science Foundation of China for financial support.

\section{References}

Lyne A.G., Manchester R.N., 1988, MNRAS 234, 477.

Qiao G.J., 1988, Vistas in Astronomy 31, 393.

Qiao G.J., 1992, Proc. IAU Collo.128, p419.

Qiao G.J., Li C.G., Li M., 1992, Proc. IAU Collo.128, p242.

Qiao G.J., Manchester R.N., Lyne A.G., Gould D.M., 1995, MNRAS 274, 572 Radhakrishnan V., Cooke D.J., 1969, Ap. Let. 3, 225.

Rankin J.M., 1983, ApJ 274, 333.

Rankin J.M., 1988, ApJ 325, 314.

Wu X.J., Manchester R.N., Lyne A.G., Qiao G.J., 1993, MNRAS 261, 630. 